\title{
The impact of adverse childhood experiences on obesity and unhealthy weight control behaviors among adolescents
}

\section{Isohookana, Reetta}

2016-11

Isohookana , R , Marttunen , M , Hakko , H , Riipinen , P \& Riala , K 2016 , ' The impact of adverse childhood experiences on obesity and unhealthy weight control behaviors among adolescents ' , Comprehensive Psychiatry , vol. 71 , pp. 17-24 . https://doi.org/10.1016/j.comppsych.2016.08.002

http://hdl.handle.net/10138/228690

https://doi.org/10.1016/j.comppsych.2016.08.002

publishedVersion

Downloaded from Helda, University of Helsinki institutional repository.

This is an electronic reprint of the original article.

This reprint may differ from the original in pagination and typographic detail.

Please cite the original version. 


\title{
The impact of adverse childhood experiences on obesity and unhealthy weight control behaviors among adolescents
}

\author{
Reetta Isohookana ${ }^{\mathrm{a}, *}$, Mauri Marttunen ${ }^{\mathrm{b}}$, Helinä Hakko ${ }^{\mathrm{c}}$, Pirkko Riipinen ${ }^{\mathrm{c}}$, Kaisa Riala ${ }^{\mathrm{a}}$ \\ ${ }^{a}$ Department of Adolescent Psychiatry, Helsinki University Central Hospital, PL 590, 00029, HUS, Finland \\ ${ }^{\mathrm{b}}$ Adolescent Psychiatry University of Helsinki, National Institute for Health and Welfare, Mannerheimintie 166, 00271 Helsinki, Finland \\ ${ }^{\mathrm{c}}$ Department of Psychiatry, Oulu University Hospital, PL 26, 90029, OYS, Finland
}

\begin{abstract}
Background: Childhood abuse and other early-life stressors associate with being overweight or obese later in life. In addition to being overweight, unhealthy weight control behaviors (e.g., vomiting, using diet pills, fasting, and skipping meals) have been shown to be common among adolescents. To our knowledge, the association between these behaviors and adverse childhood experiences (ACEs) remains unexamined.

Methods: We examined the association of ACEs to body mass index (BMI) and unhealthy weight control behaviors among 449 Finnish adolescents aged 12 to 17 years admitted to an acute psychiatric hospital unit between April 2001 and March 2006. We used the Schedule for Affective Disorders and Schizophrenia for School-Age Children Present and Lifetime (K-SADS-PL) and the European Addiction Severity Index (EuropASI) to obtain information about ACEs, psychiatric diagnoses and weight control behaviors. BMI was calculated using the weight and height measured for each adolescent upon admission.

Results: Girls who experienced sexual abuse were more likely to be obese (OR: 2.6; 95\% CI: 1.1-6.4) and demonstrate extreme weight loss behaviors (EWLB) (OR: 2.2; 95\% CI: 1.0-4.7). Among girls, parental unemployment is associated with an increased likelihood of obesity (OR: 3.5; 95\% CI: 1.2-9.6) and of being underweight (OR: 3.6; 95\% CI: 1.1-11.6). A proneness for excessively exercising was found among girls who had witnessed domestic violence (OR: 3.5; 95\% CI: 1.4-9.2) and whose parent(s) had died (OR: 5.4; 95\% CI: 1.1-27.7). Conclusion: This study showed that female adolescents with a history of traumatic experiences or difficult family circumstances exhibited an elevated likelihood of being obese and engaging in unhealthy weight control behaviors.
\end{abstract}

(C) 2016 Elsevier Inc. All rights reserved.

\section{Introduction}

Several studies have documented an association between childhood traumatic events and the development of obesity later in life [1-4]. A study from the United States found that, among a sample of adult patients seeking surgical treatment for obesity, approximately one-fifth had been exposed to sexual and physical abuse and over one-third had witnessed violence [3]. In addition, less severe forms of early-life stressors, such as separation from a mother or father or parental marital problems, were associated with an increased risk of obesity in adulthood [1].

\footnotetext{
* Corresponding author.

E-mail addresses: reetta.isohookana@gmail.com (R. Isohookana), mauri.marttunen@thl.fi (M. Marttunen), helina.hakko@oulu.fi (H. Hakko), pirkko.riipinen@oulu.fi (P. Riipinen), kaisa.riala@hus.fi (K. Riala).
}

However, only few studies have examined the influence of ACEs on being overweight or obese during adolescence [5-8]. A cross-sectional survey of secondary school students aged 13 to 16 years in the Netherlands indicated that, among boys, a history of physical abuse increased their risk for obesity by 1.8 times, while a history of sexual abuse increased their risk by 2.5 times [8]. Another study from the United States showed that cumulative ACEs doubled the risk of being overweight or obese in a pediatric population compared to children free from ACEs [6]. Emotional, sexual, and physical abuses, as well as household dysfunction such as parental alcohol or drug abuse, mental illness, and divorce, frequently fall under the term adverse childhood experiences (ACEs) [9-12].

Previous studies have established a connection between various forms of childhood abuse and excess weight or obesity $[2,13,14]$. Unhealthy weight control behaviors, such as vomiting, use of diet pills, fasting, and skipping meals are 
common among adolescents $[15,16]$. To our knowledge, the association between these behaviors and ACEs remains unstudied in adolescent populations. Therefore, this study aimed to examine the association and cumulative effect of various ACEs on becoming overweight or obese, and to determine the relation of engaging in unhealthy weight control behaviors in a clinical sample of 12 to 17 year old inpatients in Northern Finland.

\section{Materials and methods}

\subsection{Study sample}

This study represents a portion of the Study-70 project, described in detail elsewhere $[17,18]$. The original study sample consisted of 508 (300 girls and 208 boys) adolescent psychiatric inpatients aged 12 to 17 years (mean, 15.4 years) consecutively admitted for acute psychiatric hospitalization to unit 70 at Oulu University Hospital between April 2001 and March 2006. From these 508 adolescents, data on weight or height were absent for 59 individuals. Thus, our final study sample was 449 patients ( 271 girls and 178 boys). We found no difference based on age, sex, or place of residence between the 59 individuals for whom data on height or weight were missing and the final study sample. No differences existed in the prevalence of a fear of becoming obese, exercising excessively, extreme weight loss behaviors (EWLB), or binge eating in a comparison of the final study sample to those excluded from the analyses. The Ethics Committee of the University of Oulu, Finland approved the study protocol.

\subsection{Instruments}

The treating physicians or trained medical students interviewed adolescents during their hospitalization using the semi-structured Schedule for Affective Disorder and Schizophrenia for School-Age Children Present and Lifetime (K-SADS-PL) (Kaufman et al. 1997) to determine the Diagnostic and Statistical Manual of Mental Disorders, Fourth Edition (DSM-IV) diagnoses, and to identify unhealthy weight control behaviors. Using the K-SADS-PL interview, the test-retest reliability of diagnoses was rated as good to excellent, and the inter-rater agreement and concurrent validity were both high $[19,20]$. The data for the present study were collected using K-SADS-PL, with the exception of place of residence, information about parental divorce, death, and employment status, and any psychiatric and substance-related problems. This additional information was obtained from interviews using the European Addiction Severity Index (EuropASI) [21] and conducted by trained nurses on unit 70. EuropASI is a face-to-face structured interview shown to yield highly satisfactory results in terms of its reliability and validity when applied to substance-abusing populations [21].

\subsection{ACE variables}

Information on domestic violence and exposure to sexual or physical abuse was based on the screening section for post-traumatic stress disorder (PTSD) within the K-SADS-PL interview. Adolescents were asked whether they had witnessed domestic violence (the child witnessed explosive arguments involving threatened or actual harm to a parent; yes or no), experienced physical abuse from a parent(s) (bruises sustained on more than one occasion or had sustained a more serious injury; yes or no), or had experienced any form of sexual abuse (isolated or repeated incidents of genital fondling, oral sex, or vaginal or anal intercourse; yes or no).

Information regarding the employment status of a patient's mother and father was gathered during the EuropASI interview (a mother or father was defined as being employed if $\mathrm{s} / \mathrm{he}$ held a regular full- or part-time job). Data on psychiatric and substance use problems among the patient's mother or father were also obtained using EuropASI. Adolescents were asked if they felt that their mother or father had any substance abuse (alcohol, drugs, or other substances; yes or no) or psychiatric problems (yes or no) requiring treatment or if they felt their parent(s) should be treated by health professionals. Information on parental divorce (including marriage and cohabitation) and parental death (yes or no) was also obtained using the EuropASI interview.

\subsection{Body mass index (BMI)}

Body mass index (BMI; calculated as weight in kilograms divided by height in meters squared) was calculated using the weight and height for each adolescent measured by a nurse upon admission to the psychiatric ward. An adolescent was defined as normal weight if her/his BMI fell between the 25th and 85th percentiles of the age- and sex-matched Finnish population. An adolescent with a BMI under the 25th percentile was defined as underweight, an adolescent with a BMI falling between the 85th and 95th percentiles was identified as overweight, and an adolescent with a BMI falling above the 95 th percentile was identified as obese. The cut-off points were determined according to the BMI percentile values based on standard BMI reference data used in Finnish child health clinics [22].

\subsection{Fear of becoming obese}

Information about any fear of becoming obese was based on the screening section for anorexia nervosa within the K-SADS-PL interview. Adolescents were asked whether they had ever been afraid of becoming obese and how serious this fear was. Responses fell into the following categories: (1) no information; (2) not present; (3) subthreshold, indicated by an intense and persistent fear of becoming fat that defies prior weight history and/or present weight, where fears only moderately impact on behavior and/ 
or functioning; and (4) threshold, indicative of an intense and persistent fear of becoming fat severely impacting behavior and/or functioning, such as being constantly preoccupied with weight concerns or using weight loss methods once a week or more. The fear of becoming obese (yes or no) was defined as present if an adolescent fell into either the sub-threshold or threshold criterion.

\subsection{Excessively exercising}

Information regarding excessive exercise was based on the screening section for anorexia nervosa within the K-SADS-PL interview. Adolescents were asked whether they currently or previously exercised excessively. Responses fell into the following categories: (1) no information; (2) not present; (3) less than once a week; and (4) one or more times a week. The condition was present (yes or no) if an adolescent reported such behavior occurred one or more times a week.

\subsection{Extreme weight loss behavior (EWLB)}

Information regarding EWLB was based on the screening section for anorexia nervosa within the K-SADS-PL interview. Adolescents were asked whether they currently or had previously engaged in EWLB $[23,24]$, including the use of diet pills, taking laxatives or diuretics, self-induced vomiting, or drinking only non-energy fluids. Responses were categorized as follows: (1) no information; (2) not present; (3) less than once a week; or (4) one or more times a week. EWLB was defined as being present (yes or no) if an adolescent reported engaging in at least one of the behaviors at least once a week.

\subsection{Binge eating}

Information about binge eating was based on the screening section for anorexia nervosa within the K-SADS-PL interview. Adolescents were asked whether they ever engaged in uncontrollable, excessive, and rapid eating of high-energy food amounting to at least 3000 cal or more per day. Responses were categorized as follows: (1) no information; (2) not present; (3) sub-threshold, indicating such behavior occurred less than once a week; or (4) threshold, which indicated the behavior occurred at least once a week. Binge eating was defined as being present (yes or no) if the threshold criterion was fulfilled.

\subsection{Cumulative effect of ACES}

The cumulative effect of ACEs on BMI, a fear of becoming obese, excessively exercising, EWLB, and binge eating were analyzed separately among boys and girls by grouping the number of ACEs into four categories as follows: (1) one ACE, (2) two ACEs, (3) three to four ACEs, and (4) five or more ACEs.

\subsection{Psychiatric disorders}

All DSM-IV-based psychiatric disorders for the adolescents in our study were based on the K-SADS-PL interview. Psychiatric disorders were categorized into six major psychiatric diagnostic categories: (1) substance-related disorders (DSM-IV 303.9, 304.0-304.6, 304.8-304.9, $305.0,305.2-305.7,305.9)$; (2) anxiety disorders (300.00-300.02, 300.21-300.23, 300.29, 300.3, 308.3, 309.81); (3) mood disorders (296.2-296.3, 300.4, 311); (4) conduct or oppositional defiant disorders or ADHD (312.8-312.9, 313.81, 314.00-314.01, 314.9, 299.80); (5) psychotic disorders $(295,296.0,296.4-299.0,297.1-299.0$, 301.13 , 301.22); and (6) other non-specific diagnoses (292.0-292.12, 292.89, 293.82, 296.00, 296.41-296.44, 296.50, 296.61, 296.63, 296.80-296.90, 301.13, 299.80, 300.12 , 300.15, 301.22, 302.9, 307.10, 307.23, $307.50-307.51,307.60,309.00,309.21,309.28,309.30$, 309.40 , 309.90-309.91, 312.31, 312.33, 313.82, 313.90, 315.90). An adolescent was defined as belonging to a category (yes, no) if the diagnostic criteria of K-SADS-PL for that category were fulfilled. An adolescent may have several psychiatric disorders belonging to different psychiatric diagnostic categories. A total of 286 adolescents (176 girls and 110 boys) fulfilled the criteria for at least two psychiatric diagnostic categories. Adolescents with eating disorders were generally treated in another unit.

\subsection{Covariates}

In addition to psychiatric diagnoses, other covariates used in this study included the place of residence, regular smoking and the age of the adolescent. We used these covariates because previous studies have identified an association between them and weight [25-28]. Information about the adolescents' place of residence was obtained using EuropASI, and classified into two categories: (1) urban (more than 100,000 inhabitants) or (2) rural (less than 100,000 inhabitants). Data on frequent smoking (yes or no) were gathered from the screening section on tobacco use from K-SADS-PL. An adolescent was defined as a regular smoker if $\mathrm{s} /$ he smoked at least one cigarette a day.

\subsection{Statistical methods}

We assessed the statistical significance of group differences for categorical variables using the $\chi^{2}$ or Fisher's exact test, while continuous data relied on the Student's t test. The association of eight ACEs and cumulative ACEs with the outcome variables (underweight, overweight, obese, a fear of becoming obese, excessive exercising, EWLB, and binge eating) was analyzed using a binary logistic regression model after adjusting for the covariates (age at admission, place of residence, regular smoking, and psychiatric disorders). All eight ACEs were entered into the model simultaneously because of the possible relationship between any one types of ACE. We also conducted an analysis of 
missing data in which age, sex, and place of residence, as well as the prevalence of a fear of becoming obese, excessive exercising, EWLB, and binge eating, for all study subjects $(\mathrm{n}=449)$ were compared with those adolescents for whom height or weight data were missing $(n=59 ; 29$ girls and 30 boys). All statistical tests were two-tailed and we set the statistical significance level to $p<0.05$. We used IBM SPSS Statistics, version 22, for all statistical analyses.

\section{Results}

\subsection{Characteristics of the adolescents}

Table 1 presents the socio-demographic and clinical characteristics of the boys and girls in our study separately. For all ACEs, a statistically significantly higher proportion of boys than girls are exposed to parental divorce $(\mathrm{p}=$ $0.001)$, parental unemployment $(\mathrm{p}=0.021)$, and a parent's death $(\mathrm{p}=0.018)$, while a higher proportion of girls had experienced sexual abuse $(p<0.001)$ and psychiatric problems in a parent $(p=0.002)$ compared to boys. In the distribution of weight categories, obesity was more prevalent among boys than girls $(\mathrm{p}=0.008)$ but fear of becoming obese $(p<0.001)$, exercising excessively $(p<0.001)$, EWLB $(p<0.001)$, and binge eating $(p<0.001)$ were all significantly more common among girls. We also found that conduct disorders were significantly more common among boys $(\mathrm{p}<0.001)$, while affective disorders $(\mathrm{p}<0.001)$, anxiety disorders $(\mathrm{p}<0.001)$, and other diagnoses $(\mathrm{p}=$ 0.028 ) were significantly more common among girls. Further, a significantly higher proportion of boys were frequent tobacco users $(p=0.026)$ and had admitted to hospital from an urban environment $(p=0.045)$ compared to girls.

\subsection{The effect of ACEs on BMI}

Table 2 shows the logistic regression analysis of the association between various ACEs and BMI for boys and girls, respectively. We found no statistically significant differences between ACEs and BMI among boys. Girls who had experienced sexual abuse, however, exhibited a 2.6-fold increased likelihood of being obese compared to girls who had not experienced sexual abuse (OR: 2.6; 95\% CI: $1.1-6.4 ; p=0.038)$. Among girls, exposure to parental unemployment was associated with increased likelihood of obesity (OR: 3.5; 95\% CI: 1.2-9.6; $p=0.018$ ) and of being underweight (OR: 3.6; 95\% CI: 1.1-11.6; $\mathrm{p}=0.030$ ). Girls who had witnessed domestic violence, were less likely to be underweight (OR: $0.3 ; 95 \%$ CI: $0.1-0.9 ; \mathrm{p}=0.040$ ). A

Table 1

Socio-demographic and clinical characteristics of the boys and girls in a clinical sample of adolescent psychiatric inpatients.

\begin{tabular}{|c|c|c|c|}
\hline & Boys $(\mathrm{n}=178)$ & Girls $(n=271)$ & Gender difference, $\mathrm{p}$ value \\
\hline Age (in years) at admission, mean (SD) & $15.4(1.4)$ & $15.5(1.3)$ & 0.387 \\
\hline \multicolumn{4}{|l|}{ Place of residence $(\%)$} \\
\hline Urban & $94(52.8 \%)$ & $117(43.2 \%)$ & \multirow[t]{2}{*}{0.045} \\
\hline Rural & $84(47.2 \%)$ & $154(56.8 \%)$ & \\
\hline Frequent tobacco use, reported (\%) & $126(70.8 \%)$ & $164(60.5 \%)$ & 0.026 \\
\hline \multicolumn{4}{|l|}{ Psychiatric diagnoses } \\
\hline Affective disorder & $61(34.3 \%)$ & $156(57.6 \%)$ & $<0.001$ \\
\hline Anxiety disorder & $25(14.0 \%)$ & $78(28.8 \%)$ & $<0.001$ \\
\hline Substance-related disorder & $75(42.1 \%)$ & $100(36.9 \%)$ & 0.266 \\
\hline Conduct disorder & $104(58.4 \%)$ & $92(33.9 \%)$ & $<0.001$ \\
\hline Psychotic disorder & $28(15.7 \%)$ & $40(14.8 \%)$ & 0.779 \\
\hline Other diagnoses & $10(5.6 \%)$ & $32(11.8 \%)$ & 0.028 \\
\hline \multicolumn{4}{|l|}{ ACEs } \\
\hline Witnessed domestic violence & $52(29.2 \%)$ & $83(30.6 \%)$ & 0.749 \\
\hline Physical abuse & $43(24.2 \%)$ & $72(26.6 \%)$ & 0.567 \\
\hline Sexual abuse & $6(3.4 \%)$ & $62(22.9 \%)$ & $<0.001$ \\
\hline Parental unemployment & $47(36.4 \%)$ & $47(17.3 \%)$ & 0.021 \\
\hline Parental death & $19(10.7 \%)$ & $13(4.8 \%)$ & 0.018 \\
\hline Parental divorced & $108(61.0 \%)$ & $123(45.4 \%)$ & 0.001 \\
\hline Parental psychiatric problems & $22(12.4 \%)$ & $66(24.4 \%)$ & 0.002 \\
\hline Parental substance use problems & $49(27.5 \%)$ & $91(33.6 \%)$ & 0.176 \\
\hline \multicolumn{4}{|l|}{ Weight } \\
\hline Underweight (<25th percentile) & $21(11.8 \%)$ & $36(13.3 \%)$ & \multirow[t]{4}{*}{0.008} \\
\hline Normal weight (25th-85th percentile) & $80(44.9 \%)$ & $156(57.6 \%)$ & \\
\hline Overweight (85th-95th percentile) & $30(16.9 \%)$ & $40(14.8 \%)$ & \\
\hline Obese ( $>95$ th percentile) & $47(26.4 \%)$ & $39(14.4 \%)$ & \\
\hline Fear of becoming obese & $4(2.2 \%)$ & $87(32.1 \%)$ & $<0.001$ \\
\hline Excessive exercising & $0(0.0 \%)$ & $37(13.7 \%)$ & $<0.001$ \\
\hline Extreme weight loss behavior (EWLB) & $0(0.0 \%)$ & $50(18.5 \%)$ & $<0.001$ \\
\hline Binge eating & $0(0.0 \%)$ & $25(9.2 \%)$ & $<0.001$ \\
\hline Impulsivity & $51(28.7 \%)$ & $30(11.1 \%)$ & $<0.001$ \\
\hline
\end{tabular}


Table 2

Association of adverse childhood experiences (ACEs) to being underweight, overweight and obese in adolescent males and females in a clinical sample of patients aged $13-17$ years.

\begin{tabular}{|c|c|c|c|c|c|c|}
\hline \multirow[t]{3}{*}{ ACEs } & \multicolumn{2}{|c|}{ Underweight } & \multicolumn{2}{|c|}{ Overweight } & \multicolumn{2}{|c|}{ Obesity } \\
\hline & Males & Female & Males & Female & Males & Female \\
\hline & $\begin{array}{l}\text { OR }(95 \% \mathrm{CI}) \\
\mathrm{p} \text { value }\end{array}$ & $\begin{array}{l}\text { OR }(95 \% \mathrm{CI}) \\
\mathrm{p} \text { value }\end{array}$ & $\begin{array}{l}\text { OR }(95 \% \mathrm{CI}), \\
\mathrm{p} \text { value }\end{array}$ & $\begin{array}{l}\text { OR }(95 \% \mathrm{CI}), \\
\mathrm{p} \text { value }\end{array}$ & $\begin{array}{l}\text { OR }(95 \% \mathrm{CI}), \\
\mathrm{p} \text { value }\end{array}$ & $\begin{array}{l}\text { OR }(95 \% \mathrm{CI}) \\
\mathrm{p} \text { value }\end{array}$ \\
\hline Witnessed domestic violence & $1.5(0.3-7.9), 0.631$ & $0.3(0.1-0.9), \mathbf{0 . 0 4 0}$ & $1.6(0.5-5.2), 0.418$ & $0.9(0.4-2.2), 0.843$ & $1.5(0.5-4.2), 0.434$ & $0.5(0.2-1.5), 0.250$ \\
\hline Physical abuse & $0.2(0.0-1.0), 0.053$ & $1.1(0.4-3.0), 0.887$ & $0.4(0.1-1.2), 0.103$ & $0.5(0.2-1.5), 0.208$ & $0.4(0.1-1.0), 0.058$ & $1.6(0.6-4.2), 0.366$ \\
\hline Sexual abuse & $5.8(0.3-101.8), 0.232$ & $0.5(0.2-1.8), 0.314$ & $0.0(0.0-), 0.999$ & $1.5(0.6-3.5), 0.386$ & $4.0(0.5-31.3), 0.188$ & $2.6(1.1-6.4), \mathbf{0 . 0 3 8}$ \\
\hline Parental unemployment & $1.3(0.3-5.7), 0.742$ & $3.6(1.1-11.6), \mathbf{0 . 0 3 0}$ & $0.9(0.3-2.8), 0.815$ & $0.9(0.3-3.0), 0.842$ & $1.2(0.5-3.2), 0.682$ & $3.5(1.2-9.6), \mathbf{0 . 0 1 8}$ \\
\hline Parental death & $5.8(0.9-38.9), 0.071$ & $1.2(0.2-6.4), 0.851$ & $0.9(0.2-5.6), 0.941$ & $0.7(0.1-7.1), 0.773$ & $2.5(0.7-9.6), 0.180$ & $0.6(0.0-6.5), 0.644$ \\
\hline Parental divorce & $0.3(0.1-1.1), 0.066$ & $1.6(0.7-3.9), 0.308$ & $0.5(0.2-1.6), 0.246$ & $0.9(0.4-2.1), 0.790$ & $1.0(0.4-2.5), 0.937$ & $0.4(0.2-1.1), 0.072$ \\
\hline Parental psychiatric problems & $0.5(0.0-9.7), 0.673$ & 0.9 (0.3-2.6), 0.897 & $3.4(0.7-17.0), 0.133$ & $1.0(0.4-2.7), 0.921$ & $3.1(0.9-10.9), 0.083$ & $0.7(0.2-2.1), 0.529$ \\
\hline Parental substance use problems & $1.1(0.2-5.0), 0.906$ & $1.2(0.4-3.7), 0.707$ & $1.6(0.5-5.7), 0.463$ & $1.1(0.5-2.8), 0.771$ & $1.0(0.3-2.9), 0.998$ & $0.8(0.3-2.4), 0.754$ \\
\hline
\end{tabular}

The association of eight ACEs to being underweight, overweight and obese using normal BMI as reference category was assessed with a logistic regression model after controlling for age, place of residence, regular smoking and psychiatric disorders of the adolescents. All ACEs are entered to the model simultaneously.

cumulative effect of ACEs on BMI was not found in boys or girls.

\subsection{Relationship between ACEs and a fear of becoming obese and weight control behaviors}

Table 3 shows the logistic regression analysis of the association between various ACEs and a fear of becoming obese and weight control behaviors among girls. This association was only analyzed for girls, since very few male subjects reported such behaviors (Table 1). Among girls suffering from physical abuse (OR: $0.3 ; 95 \%$ CI: $0.1-0.7 ; p=0.004)$ or exposed to parental unemployment (OR: $0.4 ; 95 \%$ CI: $0.2-1.0 ; \mathrm{p}=0.041$ ), we found a decreased likelihood of a fear of becoming obese. We did, however, find an increased likelihood for exercising excessively among girls who had witnessed domestic violence (OR: 3.5; 95\% CI: 1.4-9.2, p =0.010) and whose parent(s) had died (OR: 5.4; 95\% CI: 1.1-27.7; $\mathrm{p}=0.043)$ and a decreased likelihood among girls whose parents had substance use problems (OR: $0.2 ; 95 \%$ CI:
$0.1-0.7 ; p=0.009$ ). Girls exposed to sexual abuse exhibited a 2.2-fold increased likelihood of EWLB compared with girls with no exposure to sexual abuse (OR: 2.2; 95\% CI: 1.0-4.7, $\mathrm{p}=0.042$ ). We found no association between ACEs and binge eating. No cumulative effects of ACEs were found for fear of becoming obese, exercising excessively, EWLB or binge eating.

\section{Discussion}

The main findings from our study reveal that sexual abuse and parental unemployment were associated with obesity among adolescent girls receiving psychiatric inpatient treatment. In addition, a history of sexual abuse more than doubled the likelihood for EWLB among girls. Compared to girls who reported no ACEs, girls who had witnessed domestic violence were over three times more likely to exercise excessively while girls whose parent(s) had died were more than five times more likely to exercise excessively.

Table 3

Association of adverse childhood experiences (ACEs) to fear of becoming obese, exercising excessively, extreme weight loss behavior (EWLB) and binge eating in a clinical sample of female patients aged 13-17 years.

\begin{tabular}{|c|c|c|c|c|}
\hline & Fear of becoming obese & Exercising excessively & EWLB & Binge eating \\
\hline ACEs & OR $(95 \% \mathrm{CI}), \mathrm{p}$ value & OR $(95 \% \mathrm{CI}), \mathrm{p}$ value & OR $(95 \% \mathrm{CI}), \mathrm{p}$ value & OR $(95 \% \mathrm{CI}), \mathrm{p}$ value \\
\hline Witnessed domestic violence & $1.8(0.9-3.6), 0.118$ & $3.5(1.4-9.2), \mathbf{0 . 0 1 0}$ & $1.8(0.8-4.0), 0.158$ & $1.6(0.6-4.8), 0.372$ \\
\hline Physical abuse & $0.3(0.1-0.7), \mathbf{0 . 0 0 4}$ & $1.0(0.3-2.9), 0.990$ & $0.7(0.3-1.6), 0.348$ & $2.1(0.7-6.2), 0.202$ \\
\hline Sexual abuse & $1.1(0.6-2.3), 0.709$ & $1.1(0.4-2.7), 0.916$ & $2.2(1.0-4.7), \mathbf{0 . 0 4 2}$ & $1.2(0.4-3.5), 0.753$ \\
\hline Parental unemployment & $0.4(0.2-1.0), \mathbf{0 . 0 4 1}$ & $1.7(0.6-4.9), 0.346$ & $0.8(0.3-2.1), 0.652$ & $0.9(0.3-3.1), 0.900$ \\
\hline Parental death & $1.7(0.4-6.6), 0.473$ & $5.4(1.1-27.7), \mathbf{0 . 0 4 3}$ & $2.0(0.5-9.0), 0.353$ & $3.6(0.6-21.5), 0.159$ \\
\hline Parental divorce & $1.3(0.7-2.4), 0.408$ & $0.6(0.2-1.4), 0.224$ & $0.9(0.4-1.9), 0.782$ & $1.0(0.4-2.8), 0.967$ \\
\hline Parental psychiatric problems & $1.5(0.7-2.9), 0.293$ & $0.7(0.3-2.0), 0.546$ & $1.6(0.7-3.5), 0.239$ & $0.5(0.2-1.8), 0.301$ \\
\hline Parental substance use problems & $0.5(0.3-1.1), 0.074$ & $0.2(0.1-0.7), \mathbf{0 . 0 0 9}$ & $0.5(0.2-1.1), 0.079$ & $0.7(0.2-2.4), 0.619$ \\
\hline
\end{tabular}

The association of eight ACEs to fear of becoming obese, exercising excessively, extreme weight loss behavior (EWLB) and binge eating (by using normal BMI as reference category) was assessed with a logistic regression model after controlling for age, place of residence, regular smoking and psychiatric disorders of the adolescents. All ACEs are entered to the model simultaneously. 
Our finding that sexual abuse associated with obesity concurs with a study by Keeshin et al. (2013) [7], who found that among children and adolescents admitted to an acute-care inpatient psychiatric unit those exposed to sexual abuse were 1.4 times more likely to be overweight or obese than patients who experienced no sexual abuse. Earlier studies have also suggested that adverse childhood experiences, such as sexual abuse, play a major role in obesity development later in life, potentially by inducing mental and emotional perturbations, stress, maladaptive coping responses, inflammation and metabolic disturbances [29].

Our finding regarding parental unemployment and the increased likelihood of obesity mirrors that of previous studies. Parental unemployment was previously associated with women being overweight in early adulthood in a cross-sectional retrospective study from Finland [30]. Other earlier studies have found that low childhood socio-economic status is associated with being overweight in childhood and obesity later in life [31-33]. In our study, parental unemployment was also associated with being underweight among girls. Parental unemployment may decrease family's ability to purchase healthy food, which may lead to poor nutritional choices causing weight problems in children. Furthermore, the relationship between sexual abuse and EWLB was established in a study from the United States, where sexual victimization was highly associated with purging, fasting, diet pill use, and dieting among ninth- through twelfth-grade girls [34].

Of all ACEs investigated in our study, parental death and witnessing domestic violence were found to associate with excessive exercising among girls. Witnessing inter-parental violence has been linked to children's feelings of terror, unsafety and helplessness and also to depression, anxiety, somatic complaints, and sleep disturbances [35]. Also parental death may lead to lack of parental care due to mourning and depression of the remaining parent. To our knowledge previous studies have not shown these two childhood adversities to relate specifically with excessive exercising or any restrictive eating symptomology. We find this gender specific findings important which call for further studies with larger databases and with more detailed information of quality and timing of adverse experiences of adolescents.

An unexpected finding of our study was that exposure to physical abuse and parental unemployment actually reduced the likelihood of girls fearing becoming obese. Additionally among girls, parental substance use problems negatively associated with excessive exercising. We know that a preoccupation with weight is strongly associated with levels of physical activity [36,37]. It is possible that girls living in an insecure home environment have concerns other than their weight, which may explain the decreased likelihood of excessive exercising. Another explanation may be that obese girls living with unemployed parents no longer worry about their weight or fear of becoming overweight as girls in other weight categories do. This explanation parallels that from a
French study that found that overweight girls were more depressed than obese girls [38].

In this study, we found no association between ACEs and BMI among boys. In our study sample the prevalence of obesity was higher among boys compared to girls, which at least in part may be explained by well-known gender differences in the prevalence of psychiatric diagnoses and their comorbidity. It is known that eating disorders are more common among girls, and boys suffer more often instead from disruptive disorders in adolescence [39]. It is also possible that boys react to different symptoms than girls when they encounter the adverse life events and this could explain why no associations to the BMI or weight loss behaviors were found. On the other hand, an adverse experience, like isolation from the peer group and exclusion from the yard games, could lead to decline in physical activity and weight gain in boys, which could be interesting to examine in the future studies.

The strengths of our study include its use of the semi-structured K-SADS-PL and structured EuropASI interview protocols to collect all of the variables and covariates used, tools with a documented high reliability and validity. A nurse measured patient weight and height upon admission to the hospital and our calculation of the BMI categories (underweight, overweight, obese, and normal weight) used an age- and sex-matched Finnish population for reference purposes. Our study consisted of a consecutive sample of adolescents requiring acute, closed-ward psychiatric inpatient care from a geographically large area in northern Finland.

One limitation of our study is that the K-SADS-PL interview does not define the exact timing of adverse life events or the appearance of symptoms. However, we may assume that changes in BMI, weight concerns and weight control behaviors occurred following exposure to ACEs. A weakness of self-reporting measures is that not all adolescents will be able to accurately or willingly recall or describe adverse life events. It is also clear that we could not exclude all other adverse life events, such as bullying or serious illness, or dietary and genetic factors which may possibly affect to the outcomes. Since the study sample consisted of adolescent patients in a psychiatric unit, our results may not be generalizable to adolescents in the general population. Despite the relatively large study sample, the small number of cases recorded for some subgroup analyses may have resulted in insufficient statistical power and type II errors. Given the many statistical comparisons performed in our study, the additional risk of chance findings and type I error cannot be excluded. Our study is exploratory in nature that needs to be replicated in future studies.

Our study demonstrates that adolescent girls exposed to traumatic live events or living through difficult family circumstances have an elevated likelihood of becoming obese and engaging in unhealthy weight control behaviors. An assessment of an adolescent psychiatric patient's exposure to traumatic life events and adverse family 
circumstances may also help to identify cases requiring intervention to prevent obesity later in life. In turn, for example school health care could pay attention to overweight adolescents and their possible adverse life events as a part of weight monitoring to steer young people to get psycho-social support and counseling if necessary.

\section{Acknowledgment}

This study received support from the Lastenlinna Foundation, the Psychiatry Research Foundation, and the Jalmari and Rauha Ahokkaan Foundation, for which the authors are very grateful.

\section{References}

[1] D’Argenio A, Mazzi C, Pecchioli L, Di Lorenzo G, Siracusano A, Troisi A. Early trauma and adult obesity: is psychological dysfunction the mediating mechanism. Physiol Behav 2009;98:543-6.

[2] Gunstad J, Paul R, Spitznagel M, Cohen R, Williams L, Kohn M, et al. Exposure to early life trauma is associated with adult obesity. Psychiatry Res 2006;142:31-7.

[3] Sansone R, Schumacher D, Wiederman M, Routsong-Weicher L. The prevalence of childhood trauma and parental caretaking among gastric surgery candidates. Eat Disord 2008;16:117-27.

[4] Boynton-Jarret R, Rosenberg L, Palmer J, Boggs D, Wise L. Child and adolescent abuse in relation to besity in adulthood: the black women's health study. Pediatrics 2012;130(2):245-53.

[5] Pinhas-Hamiel O, Modan-Moses D, Herman-Raz M, Reichman B. Obesity in girls and penetrative sexual abuse in childhood. Acta Paediatr 2009;98:144-7.

[6] Burke N, Hellman J, Scott B, Weems C, Carrion V. The impact of adverse childhood experiences on urban pediatric population. Child Abuse Negl 2011;35:408-13.

[7] Keeshin B, Luebbe A, Strawn J, Saldana S, Wehry A, Delbello M. Sexual abuse is associated with obese children and adolescents admitted for psychiatric hospitalization. J Pediatr 2013;163:154-9.

[8] Veldwijk J, Proper K, Hoeven-Mulder H, Bemelmans W. The prevalence of psysical, sexual and mental abuse among adolescents and the association with BMI staus. BMC Public Health 2012;840(12): 1471-2458.

[9] Dube S, Anda R, Felitti V. Childhood abuse, household dysfunction, and the risk of attempted suicide throughout the life span: findings from the adverse childhood experiences study. JAMA 2001;286(24):3089-96.

[10] Anda R, Croft J, Felitti V, Nordenberg D, Giles W, Williamson D. Adverse childhood experiences and smoking during adolescence and adulthood. JAMA 1999;282(17):1652-8.

[11] Chapman D, Whitfield C, Felitti V, Dube S, Edwards V, Anda R. Adverse childhood experiences and the risk of depressive disorders in adulthood. J Affect Disord 2004;82:217-25.

[12] Schilling E, Aseltine R, Gore S. Adverse childhood experiences and mental health in young adults. BMC Public Health 2007;7:30, http:/ dx.doi.org/10.1186/1471-2458-7-30.

[13] Fuemmeler B, Dedert E, McClernon F, Beckham J. Adverse childhood events are associated with obesity and disordered eating: results from a U.S. population-based survey of young adults. J Trauma Stress 2009; 22(4):329-33.

[14] Roenholt S, Beck N, Karslberg S, Elklit A. Post-traumatic stress symptoms and childhood abuse categories in a national representative sample for a specific age group: associations to body mass index. Eur $\mathrm{J}$ Psychotraumatol 2012:3, http://dx.doi.org/10.3402/ejpt.v3i0.17188.

[15] Utter J, Denny S, Percival T, Crengle S, Ameratunga S, Dixon R, et al. Prevalence of weight-related concerns and behaviours among
New Zealand young people. J Paediatr Child Health 2012;48(11): 1021-8.

[16] Laakso E, Hakko H, Räsänen P, Riala K. Suicidality and unhealthy weight control behaviors among female underaged psychiatric inpatients. Compr Psychiatry 2013;54(2):117-22.

[17] Isohookana R, Riala K, Hakko H, Räsänen P. Adverse childhood experiences and suicidal behavior of adolescent psychiatric inpatients. Eur Child Adolesc Psychiatry 2013;22:13-22.

[18] Ilomäki R, Södervall J, Ilomäki E, Hakko H, Räsänen P. Drug-dependent boys are more depressed compared to girls: a comorbidity study of substance dependence and mental disorders. Eur Addict Res 2008;14(3):161-8.

[19] Ambrosini P. Historical development and present status of the schedule for affective disorders and schizophrenia for school-age children (K-SADS). J Am Acad Child Adolesc Psychiatry 2000;39(1):49-58.

[20] Kaufman J, Birmaher B, Rao U, Flynn C, Moreci P, Williamson D. Schedule for affective disorders and schizophrenia for school-age childrenpresent and lifetime ersion (K-SADS-PL): initial reliability and validity data. J Am Acad Child Adolesc Psychiatry 1997;36(7):980-8.

[21] Kokkevi A, Hartgers C. European adaptation of a multidimensional assessment instrument for drug and alcohol dependents. Eur Addict Res 1995;1:208-10.

[22] Wei Y, Pere A, Koenker R, He X. Quantile regression methods for reference growth charts. Stat Med 2009;25:1369-82.

[23] Hutchinson D, Rapee R. Do friends share similar body image and eating problems? The role of social networks and peer influences in early adolescence. Behav Res Ther 2007;45:1557-77.

[24] Liechty J, Lee M. Longitudinal predictors of dieting and disordered eating among young adults in the U.S. Eat Disord 2013;46(8):790-800.

[25] Dudzinska M, Tarach J, Zwolak A, Kurowska M, Malicka J, Smolen A, et al. Type 2 diabetes mellitus in relation to place of residence: evaluation of selected aspects of socio-demographic status, course of diabetes and quality of life - a cross-sectional study. Ann Agric Environ Med 2013;20(4):869-74.

[26] Jong-Hyuck K, Wi-Young S. Association of smoking frequency and cigarette consumption with obesity in Korean adolescents. Bratisl Lek Listy 2012;113(10):599-603.

[27] Britvik D, Maric N, Doknic N, Pekic S, Andric S, Jasovic-Gasic M, et al. Metabolic issues in psychotic disorders with the focus on first-episode patients: a review. Psychiatr Danub 2013;25(4):410-5.

[28] Guedes D, Almeida F, Neto J, Maia M, Tolentino T. Low body weight/ thinness overweight and obesity of children and adolescents from a Brazilian region of low economic status. Rev Paul Pediatr 2013;31(4): 437-43.

[29] Hemmingsson E, Johansson K, Revnisdottir S. Effect of childhood abuse on adult obesity: a systematic review and meta-analysis. Obes Rev 2004;15(11):882-93.

[30] Kestilä L, Rahkonen O, Martelin T, Lahti-Koski M, Koskinen S. Do childhood social circumstances affect overweight and obesity in early adulthood. Public Health 2009;37(2):206-19.

[31] Tamayo T, Christian H, Rathmann W. Impact of early psychosocial factors (childhood socioeconomic factors and adversities) on future risk of type 2 diabetes, metabolic disturbances and obesity: a systematic review. BMC Public Health 2010, http://dx.doi.org/ 10.1186/1471-2458-10-525.

[32] Noh J, Kim Y, Oh I, Kwon Y. Influences of socioeconomic factors on childhood and adolescent overweight by gender in Korea: crosssectional analysis of nationally representative sample. BMC Public Health 2014;14:324, http://dx.doi.org/10.1186/1471-2458-14-324.

[33] Singh G, Siahpush M, Kogan M. Rising social inequalities in US childhood obesity, 2003-2007. Ann Epidemiol 2010;20:40-52.

[34] Thompson K, Wonderlich S, Crosby R, Mitchell J. Sexual victimization and adolescent weight regulation practices: a test across three community based samples. Child Abuse Negl 2001;25:291-305.

[35] Klostermann K, Kelley M. Alcoholism and intimate partner violence: effects on children's psychosocial adjustment. Environ Res Public Health 2009;6(12):3156-68. 
[36] Davis C, Brewer H, Ratusny D. Behavioral frequency and psychological commitment: necessary concepts in the study of excessive exercising. J Behav Med 1993;16(6):611-28.

[37] De Young K, Anderson D. The importance of the function of exercise in the relationship between obligatory exercise and eating and body image concerns. Eat Behav 2010;11(1):62-4.
[38] Revah-Levy A, Speranza M, Barry C, Hassler C, Gasquet I, Moro M, et al. Association between body mass index and depression: the "fat and jolly" hypothesis for adolescents girls. BMC Public Health 2011;11:649, http://dx.doi.org/10.1186/1471-2458-11-649.

[39] Herpertz-Dahlmann B, Buhren K, Remschmidt H. Growing up is hard, mental disorders in adolescence. Dtsch Arztebl Int 2013;110(25):432-40. 